\title{
Differentiation Syndrome with Severe Abdominal Pain During Induction Treatment of Acute Promyelocytic Leukemia: A Case Report
}

\author{
Haimeng Yan' \\ Donghua $\mathrm{He}^{\prime}$ \\ Weijia Huang' \\ Fei Chen ${ }^{1,2}$ \\ Jingsong $\mathrm{He}^{1}$ \\ Zhen Cai ${ }^{1,3,4}$ \\ Yi Zhao'
}

'Bone Marrow Transplantation Center, The First Affiliated Hospital, Zhejiang University School of Medicine, Hangzhou, People's Republic of China; ${ }^{2}$ Department of Hematology, Haining People's Hospital, Haining, People's Republic of China; ${ }^{3}$ Institute of Hematology, Zhejiang University, Hangzhou, Zhejiang, People's Republic of China; ${ }^{4}$ Zhejiang Laboratory for Systems \& Precision Medicine, Zhejiang University Medical Center, Hangzhou, 3I I I2I, People's Republic of China
Correspondence: Yi Zhao; Zhen Cai Bone Marrow Transplantation Center, Zhejiang University School of Medicine, Qingchun Road 79, Hangzhou, 310009 ,

People's Republic of China

Email zhaoyi999@zju.edu.cn;

caiz@zju.edu.cn
Background: Acute promyelocytic leukemia differentiation syndrome (APL DS) is a common and severe complication seen in patients with APL treated with all-trans retinoic acid (ATRA) and/or arsenic trioxide (ATO). The presenting symptoms of APL DS are diverse, and rare symptoms are easy to be misdiagnosed. Therefore, it is very crucial to identify DS from uncommon signs to avoid delay in treatment.

Case Presentation: Here, we report a patient of APL who developed severe abdominal pain during ATRA and ATO therapy, with increasing leukocyte count. Organic diseases were firstly excluded, and empiric treatment for DS was adopted. The abdominal pain was gradually relieved and the patient eventually achieved complete remission.

Conclusion: This case history suggests that APL DS may manifest as severe abdominal pain, and the early identification of DS and immediate treatment could improve the prognosis of patients.

Keywords: acute promyelocytic leukemia, all-trans-retinoic acid, arsenic trioxide, differentiation syndrome, abdominal pain, case report

\section{Introduction}

The survival rate of acute promyelocytic leukemia (APL) has been significantly improved since all-trans-retinoic acid (ATRA) and arsenic trioxide (ATO) were introduced into chemotherapeutic regimens. ${ }^{1}$ However, some severe treatmentrelated complications, such as intracranial bleeding, differentiation syndrome (DS), and other ATRA- and ATO-related toxicities, limit the therapeutic effect. ${ }^{2}$

DS often occurs in APL patients when they are undergoing induction therapy with ATRA and/or ATO. ${ }^{3}$ Presenting symptoms of APL DS are diverse but are frequently characterized by unexplained fever, dyspnea with pulmonary infiltrates or pleural or pericardial effusion, weight gain, hypotension and acute renal failure. ${ }^{4}$ It is worth noting that many cases of APL DS were reported with rare symptoms, which make the diagnosis and treatment challenging. Here, we report the case of a young patient with APL who developed DS characterized by severe abdominal pain during induction therapy with ATRA and ATO.

\section{Case Presentation}

A 17-year-old male student presented with a 3-day history of bleeding from the gums prior to admission. At admission, the white blood cell (WBC) count was $12.67 \times 10 \mathrm{E} 9 / \mathrm{L}$, hemoglobin (HB) was $137 \mathrm{~g} / \mathrm{L}$, and platelet (PLT) count was 6x10E9/L. Bone marrow 
aspiration showed $86 \%$ promyelocytic granulocytes, and leukemia immunotyping was consistent with the diagnosis of APL (high risk). Flow cytometry showed that CD13, CD33 and CD117 were highly expressed, CD56 was weakly expressed, and CD34, HLA-DR, CD11b, CD14 and CD64 were not expressed. Chromosome t $(15 ; 17)(\mathrm{q} 24 ; \mathrm{q} 21)$ and PML/RAR alpha were also confirmed, and he was treated with ATRA (20 mg/day) and ATO (10 mg/day). At the same time, he received idarubicin (IDA, $5 \mathrm{mg} /$ day) to decrease the $\mathrm{WBC}$ count. On day 4 , the WBC count was $7.13 \times 10 \mathrm{E} 9 / \mathrm{L}$, and IDA was discontinued. On day 12 after ATRA and ATO initiation, the patient complained of slight headache without any other discomforts. The WBC count was 14.44x10E9/L. Considering the risk of DS, ATRA was discontinued, and cytarabine (Ara$\mathrm{C}, 50 \mathrm{mg} /$ day) was administered. On day 14, he developed severe and persistent total abdominal pain, which was unbearable, with no fever or dyspnea. Physical examination revealed abdominal tension and tenderness. The WBC count was 19.66x10E9/L, HB was $98 \mathrm{~g} / \mathrm{L}$, PLT count was 50x10E9/L, fibrinogen was $1.91 \mathrm{~g} / \mathrm{L}$, ALT was $162 \mathrm{U} / \mathrm{L}$, AST was $73 \mathrm{U} / \mathrm{L}$, total bile acid was $15.1 \mu \mathrm{mol} / \mathrm{L}$, and D-dimer was $2500.00 \mu \mathrm{g} /$ L (normal range: $0-700 \mu \mathrm{g} / \mathrm{L}$ ). Prothrombin time bilirubin and potassium were normal. Tramadol and anisodamine could only temporarily relieve the pain. Enhanced abdominal CT suggested that there was more gas and contents in part of the intestines and little effusion in the pelvic cavity, showing no evidence of intestinal perforation, urinary calculi or arteriovenous thrombosis (Figure 1). As APL DS was suspected, ATO was discontinued, and methylprednisolone (40 $\mathrm{mg} \mathrm{q} 12 \mathrm{~h}$ for 3 days) was administered. IDA ( $5 \mathrm{mg} /$ day) was also adopted for 2 days. At the same time, the patient was given supportive

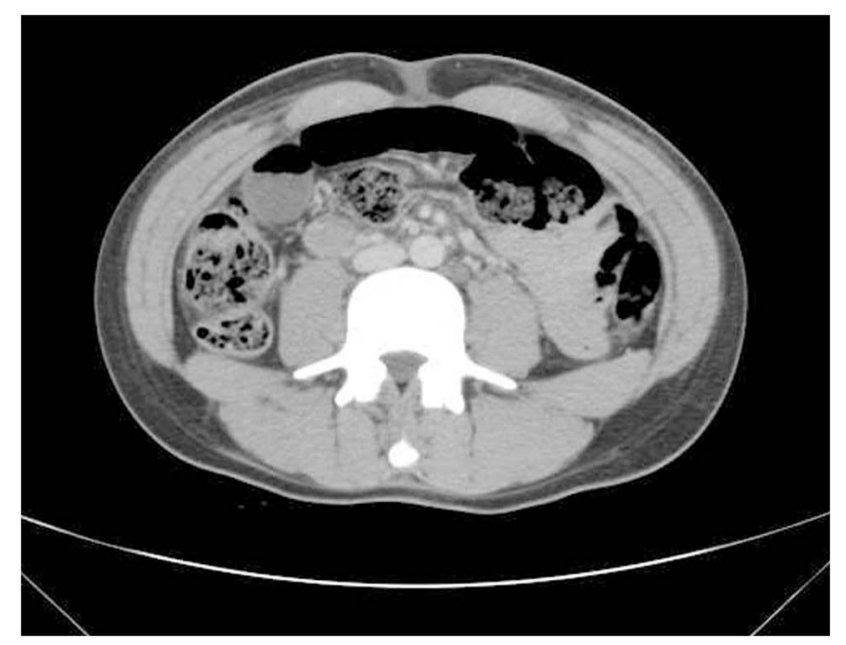

Figure I Enhanced abdominal CT taken on day 14. Part of the intestine was dilated and there was gas and fluid accumulation, and the gas-liquid level was visible. treatment, including fasting, gastrointestinal decompression and stomach protection. The symptoms of abdominal pain gradually relieved over days. The count of WBC reached a maximum of $41.45 \times 10 \mathrm{E} 9 / \mathrm{L}$, IDA ( $5 \mathrm{mg} /$ day for 2 days) was then adopted. The WBC count gradually decreased. On day 25, the patient's blood cells had recovered after supportive treatment, and he received ATRA (20 mg/day) to continue the induction therapy. During treatment with ATRA, no APL DS-like manifestations or abdominal pain occurred. On day 33, a subsequent bone marrow examination showed that he had achieved complete remission associated with incomplete recovery of blood cells (CRi). The clinical course is illustrated in Figure 2.

\section{Discussion}

We present a unique case report of APL DS with severe abdominal pain as the main manifestation. This patient had three features: (a) the development of severe abdominal pain during ATRA and ATO therapy, with increasing leukocyte count; (b) treatment, including stopping ATRA and ATO and administering methylprednisolone, was effective, confirming the presumptive diagnosis of DS; and (c) the second administration of ATRA did not lead to abdominal discomfort.

Acute promyelocytic leukemia differentiation syndrome (APL DS) is an adverse event that occurs during induction therapy with ATRA in APL patients. ${ }^{5}$ The pathogenesis of DS is complex and not entirely clarified. ${ }^{6}$ It has been speculated that ATRA leads to the release of cytokines by differentiating myeloid cells and endothelial damage. Based on these pathophysiologic mechanisms, APL DS presents in diverse and nonspecific symptoms, which make the diagnosis of DS challenging. In addition to the most common signs, rare symptoms such as diffuse alveolar hemorrhage, ${ }^{7}$ Sweet's syndrome ${ }^{8}$ and ocular manifestations ${ }^{9}$ have also been reported. It is necessary to identify DS when patients present with rare symptoms. The early identification of DS and immediate treatment with steroids appear to reduce DS-related mortality. In our case, the main manifestation of APL DS was severe abdominal pain, and effective methylprednisolone therapy supported the diagnosis of DS.

Many cases of abdominal complications that occurs during induction therapy in APL patients have been reported. Bhargava $\mathrm{R}$ described a patient with APL who developed ileal perforation because of the promyelocytic deposits in the intestine on day 18 from ATRA administration. $^{10}$ Severe stomatitis and ileocecal 


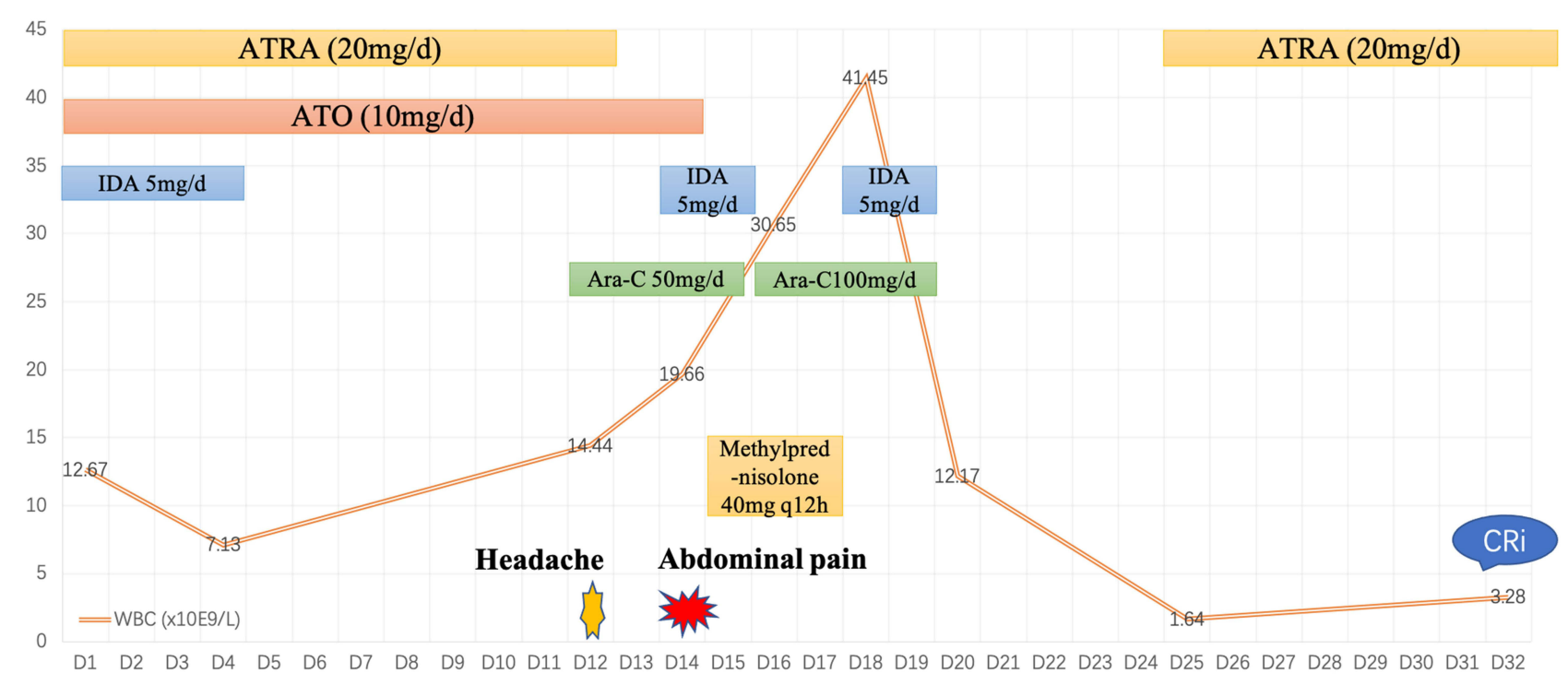

Figure 2 Clinical course of the patient.

Abbreviations: ATRA, all-trans retinoic acid; ATO, arsenic trioxide; IDA, idarubicin; Ara-C, cytarabine; WBC, white blood cell; CRi, complete remission associated with incomplete recovery of blood cells.

perforation have also been reported in APL on day 48 of ATRA discontinuous administration. ${ }^{11}$ These cases indicated that ATRA was related with the function of intestine in some way. In our case, the patient demonstrated severe abdominal pain during ATRA and ATO therapy, with increasing leukocyte count. Infection was excluded, as the patient had no skin, oral and anal ulcers, no symptoms of coughing and expectoration. C-reactive protein and procalcitonin were normal, and $\mathrm{CT}$ in the lungs did not indicate infection. The patient had no history of gastrointestinal disease. When the abdominal pain occurred, serum lipase and amylase were normal. We further excluded the diagnosis of intestinal perforation, urinary calculi, arteriovenous thrombosis, acute pancreatitis, intestinal vasculitis and necrosis by the examination of enhanced abdominal CT. The enhanced CT also showed increased gas and contents in intestines, indicating the possibility of paralytic incomplete intestinal obstruction. Notably, a paralytic ileus has been reported in relapsed APL on day 15 of ATRA treatment. ${ }^{12}$ We therefore assumed the possibility that severe abdominal pain was related with DS.

This case suggests that APL DS may mainly manifest as severe abdominal pain without any other typical symptoms. When a patient with APL undergoing induction therapy develops severe abdominal pain, DS as well as acute abdomen should be kept in mind. As ATRA treatment experience increases and more side-effect profiles are reported, the definition of DS may broaden.

\section{Abbreviations}

APL, acute promyelocytic leukemia; DS, differentiation syndrome; ATRA, all-trans retinoic acid; ATO, arsenic trioxide; WBC, white blood cell; HB, hemoglobin; PLT, platelet; IDA, idarubicin; Ara-C, cytarabine; Cri, incomplete recovery of blood cells.

\section{Ethics Approval}

The studies involving human participants were reviewed and approved by The First Affiliated Hospital, Zhejiang University School of Medicine, Hangzhou, China.

\section{Consent Statement}

The patient has provided informed consent for publication of the case details and images.

\section{Acknowledgments}

The authors thank the patient for allowing for his case to be presented.

\section{Funding}

The study was supported by the Medical and Health Science and Technology Project of Zhejiang Province (Project No. 2021KY143).

\section{Disclosure}

The authors declare that there are no conflicts of interest related to this report. 


\section{References}

1. Sanz MA, Fenaux P, Tallman MS, et al. Management of acute promyelocytic leukemia: updated recommendations from an expert panel of the European LeukemiaNet. Blood 2019;133(15):1630-1643. doi:10.1182/blood-2019-01-894980

2. Stahl M, Tallman MS. Acute promyelocytic leukemia (APL): remaining challenges towards a cure for all. Leuk Lymphoma. 2019;60 (13):3107-3115. doi:10.1080/10428194.2019.1613540

3. Stahl M, Tallman MS. Differentiation syndrome in acute promyelocytic leukaemia. Br J Haematol. 2019;187(2):157-162. doi:10.1111/ bjh. 16151

4. Montesinos P, Bergua JM, Vellenga E, et al. Differentiation syndrome in patients with acute promyelocytic leukemia treated with all-trans retinoic acid and anthracycline chemotherapy: characteristics, outcome, and prognostic factors. Blood 2009;113(4):775-783. doi:10.1182/blood-2008-07-168617

5. Abedin S, Altman JK. Acute promyelocytic leukemia: preventing early complications and late toxicities. Hematology Am Soc Hematol Educ Prog. 2016;2016(1):10-15. doi:10.1182/asheducation-2016.1.10

6. Sanz MA, Montesinos P. How we prevent and treat differentiation syndrome in patients with acute promyelocytic leukemia. Blood 2014;123(18):2777-2782. doi:10.1182/blood-2013-10-512640
7. Nicolls MR, Terada LS, Tuder RM, Prindiville SA, Schwarz MI. Diffuse alveolar hemorrhage with underlying pulmonary capillaritis in the retinoic acid syndrome. Am J Respir Crit Care Med. 1998;158 (4):1302-1305. doi:10.1164/ajrccm.158.4.9709085

8. Park CJ, Bae YD, Choi JY, et al. Sweet's syndrome during the treatment of acute promyelocytic leukemia with all-trans retinoic acid. Korean J Intern Med. 2001;16(3):218-221. doi:10.3904/kjim.2001.16.3.218

9. Newman AR, Leung B, Richards A, Campbell TG, Wellwood J, Imrie FR. Two cases of differentiation syndrome with ocular manifestations in patients with acute promyelocytic leukaemia treated with all-trans retinoic acid and arsenic trioxide. Am J Ophthalmol Case Rep. 2018;9:106-111. doi:10.1016/j.ajoc.2018.01.026

10. Bhargava R, Dolai TK, Singhal D, Kumar R, Pathak P. Retinoic acid syndrome after first dose of ATRA and ileal perforation secondary to promyelocytes infiltration. Leuk Res. 2008;32(6):997-998. doi:10.1016/j.leukres.2007.09.021

11. Kimura K, Takeuchi M, Hasegawa N, et al. [Severe stomatitis and ileocecal perforation developed after all-trans retinoic acid monotherapy in an HLA-B51-positive patient with acute promyelocytic leukemia]. Rinsho Ketsueki. 2016;57(6):765-770. doi:10.11406/rinketsu.57.765

12. Yamamoto Y, Morii T, Kimura H. Paralytic ileus occurring during treatment with all-trans retinoic acid for acute promyelocytic leukemia. Int J Hematol. 2004;79(2):198-199. doi:10.1532/IJH97.03126

\section{Publish your work in this journal}

The Journal of Pain Research is an international, peer reviewed, open access, online journal that welcomes laboratory and clinical findings in the fields of pain research and the prevention and management of pain. Original research, reviews, symposium reports, hypothesis formation and commentaries are all considered for publication. The manuscript management system is completely online and includes a very quick and fair peer-review system, which is all easy to use. Visit http:// www.dovepress.com/testimonials.php to read real quotes from published authors. 